\title{
Accuracy of Colposcopically Guided Diagnostic Methods for the Detection of Cervical Intraepithelial Neoplasia
}

\section{Treffsicherheit der kolposkopisch gestützten Differenzialdiagnostik zervikaler intraepithelialer Neoplasien}

Authors

Affiliation
K. Müller, P. Soergel, P. Hillemanns, M. Jentschke

Department of Gynaecology and Obstetrics, Hannover Medical School, Hannover

\section{Key words \\ - virus infection \\ - cervical cancer \\ - cervical intraepithelial neoplasia (CIN) \\ - cervix \\ Schlüsselwörter \\ - Virusinfektion \\ - Gebärmutterhalskrebs \\ - zervikale intraepitheliale Neoplasie \\ - Gebärmutterhals}

Deutsche Version unter: www.thieme-connect.de/ ejournals/gebfra

received 20.9.2015

revised 14.11.2015

accepted $\quad$ 15.12.2015

\section{Bibliography}

Dol http://dx.doi.org/ 10.1055/s-0041-111504

Geburtsh Frauenheilk 2016; 76: 182-187 @ Georg Thieme Verlag KG Stuttgart · New York . ISSN 0016-5751

\section{Correspondence}

Dr. med. Matthias Jentschke

Department of Gynaecology and Obstetrics

Hannover Medical School

Carl-Neuberg-Straße 1

30625 Hannover

Jentschke.Matthias@

mh-hannover.de

\section{Abstract}

$\nabla$

Introduction: Many factors can affect the accuracy of colposcopically guided biopsy, endocervical curettage (ECC) and differential cytology, all of which are standard, minimally invasive procedures used to detect cervical intraepithelial neoplasia.

Method: All conizations carried out between 2007 and 2013 in the gynecological department of Hannover Medical School were retrospectively reviewed. The agreement between colposcopic diagnosis and histology was evaluated retrospectively. The analysis included 593 complete datasets out of a total of 717 cases treated.

Results: The overall agreement was $85.5 \%$; the accuracy was significantly higher $(p=0.029)$ when three biopsy specimens were taken rather than just one. The agreement between diagnosis and histological findings from conization was highest for women $<30$ years $(90.7 \%)$ and lowest for women $>50$ years $(72.1 \% ; p=0.008)$. The agreement between preoperative differential cytology and histology results after conization was $86.7 \%$ and improved as patient age increased $(p=0.035)$. The agreement between ECC findings and the results of conization was only $49.1 \%$ irrespective of patient age, transformation zone or the patient's menopausal status.

Conclusion: The accuracy of colposcopically guided biopsy appears to increase when three biopsy specimens are taken and is particularly high for younger patients. Differential cytology was also found to be highly accurate and is particularly useful for patients aged more than 50 years. The accuracy of ECC was significantly lower; however ECC can provide important additional information in selected cases.

\section{Zusammenfassung \\ $\nabla$}

Einleitung: Die Abklärungskolposkopie mit Biopsieentnahme, endozervikaler Kürettage (ECC) und Differenzialzytologie als Goldstandard in der minimalinvasiven Diagnostik zervikaler intraepithelialer Neoplasien wird in ihrer Aussagekraft durch verschiedene Faktoren beeinflusst.

Methodik: Die Übereinstimmung der kolposkopischen Diagnose mit der endgültigen Histologie wurde retrospektiv anhand aller zwischen 2007 bis 2013 durchgeführten Konisationen in der Frauenklinik der Medizinischen Hochschule Hannover ausgewertet. Von insges. 717 ermittelten Fällen konnten 593 vollständige Datensätze in die Auswertung aufgenommen werden.

Ergebnisse: Insgesamt betrug die Übereinstimmung 85,5\% mit signifikant höherer Treffsicherheit $(p=0,029)$ bei der Entnahme von 3 Biopsien vs. 1 Biopsie. Die Übereinstimmung war am höchsten bei Frauen < 30 Jahren (90,7\%) und am geringsten $>50$ Jahren $(72,1 \%$; $=0,008)$. Der differenzialzytologische Abstrich ergab eine Übereinstimmung von 86,7\% mit zunehmender Treffsicherheit bei höherem Alter ( $p=0,035)$. Die ECC hatte eine Übereinstimmung von nur 49,1\% mit dem Konisat unabhängig von Alter, Transformationszone oder Menopausenstatus.

Schlussfolgerung: Die Treffsicherheit der kolposkopisch gestützten Biopsie scheint sich durch die Entnahme von 3 Proben steigern zu lassen und besitzt eine sehr hohe Aussagekraft vor allem für jüngere Patientinnen. Auch die Differenzialzytologie zeigt eine hohe Treffsicherheit und kann insbesondere bei Patientinnen über 50 Jahren von Nutzen sein. Die ECC liegt mit ihrer Treffsicherheit deutlich darunter, kann aber in ausgewählten Fällen wichtige zusätzliche Informationen liefern. 


\section{Introduction}

\section{$\nabla$}

Differential colposcopy with colposcopically guided biopsy is considered the gold standard of all minimally invasive diagnostic procedures used to detect cervical intraepithelial neoplasia (CIN) [1]. The colposcope provides an enlarged view of the ectocervix in different magnifications, allowing physicians to look for dysplastic changes (punctation, mosaic-like patterns) after a prior application of a $5 \%$ acetic acid solution. For the diagnosis, biopsy specimens are taken from abnormal areas and examined histologically [2]. According to the guidelines, findings of CIN 1 and 2 only require regular subsequent colposcopic check-ups. CIN 3 (and persistent CIN 2) should be treated surgically. The surgical method of choice is loop or laser conization [1].

Studies have already reported a high correlation between colposcopy findings and the histological results of cervical biopsies. In a Romanian study the agreement between differential colposcopy and histology was $78.5 \%$ for CIN 1 lesions, $84 \%$ for CIN 2 lesions and $88.6 \%$ for CIN 3 lesions. The overall sensitivity of differential colposcopy without biopsy was reported to be $83.6 \%$ [3]. Another study reported a correlation of $88.3 \%$ within one histological grade [4]. Other studies have also investigated the correlation between histological assessment of biopsy findings and the final histological result after conization. Studies have reported complete agreement in between 45 and $89.6 \%$ of cases. Agreement within one histological grade was present in between 74 and $89.9 \%$ of cases [5-11].

The crucial issue is to determine which conditions and factors can influence the accuracy of biopsy findings. One study has already shown that patient age can be a factor which will affect the accuracy of the biopsy [6]. This raises the questions whether the correlation between biopsy findings and final histology will be affected if a greater number of biopsies is taken and whether there is a connection between the correct histology and the experience of the colposcopist.

Other important methods used to obtain a differential diagnosis of CIN in addition to biopsy include (repeat) cytology and endocervical curettage (ECC). The agreement between cytology results and histology results based on biopsy has been reported as $52 \%$ [12], with an agreement of $37 \%$ reported for low-grade squamous epithelial lesions (LSIL) and $76 \%$ for high-grade squamous epithelial lesions (HSIL) [13]. The use of ECC has been discussed for those cases where colposcopy findings cannot be adequately assessed, for example women with type 3 transformation zone [14-17].

This is the context in which the conizations carried out between 2007 and 2013 in the gynecological department of Hannover Medical School were retrospectively reviewed. In addition to the factors described above which can affect the accuracy of colposcopically guided biopsies, the study also aimed to compare the extent of agreement between cytology and ECC with the final histological results obtained from conization.

\section{Material and Method}

$\nabla$

\section{Patient population}

For the retrospective analysis, all conizations carried out in the gynecological department of Hannover Medical School between January 1, 2007 and December 31, 2013 were identified. 124 patients out of a total of 717 identified cases who underwent conization in this period were excluded from the study. 91 patients had not had either biopsy or ECC preoperatively. In four cases there was no information available on the number of biopsies taken. Nine patients did not undergo conization because of dysplastic changes but for other reasons, including pre-existing cervical stenosis or ectopia resistant to treatment. In 14 cases treatment consisted of repeat conization, usually after incomplete initial resection (R1 status) which led to direct re-conization without prior biopsy. The histological results from the re-conization were either the same as after the first conization or showed no detectable dysplasia. A further 6 cases could not be assessed, either because data were lacking or because they had been listed twice.

\section{Data collection}

The data were obtained from patient records (patient admission forms, records of examinations, surgery reports and pathological findings). Data were collected from the last preoperative examination with biopsy performed prior to conization. Information on the patient's transformation zone, HPV status, and cytological and histological findings (biopsy/ECC) were included. The classification of cytological findings was done according to the Munich II nomenclature [18]; the classification of histological findings from biopsies/ECC/conization was done in accordance with the three-step CIN classification system (CIN 1-3, adenocarcinoma in situ, invasive carcinoma) [19]. Data about the examiner and the surgeon were also included, and they were classified according to their clinical experience (1-2 years, 2-5 years, more than 5 years).

In most cases cervical assessment was done by liquid-based cytology (Thinprep, Hologic Deutschland GmbH, Wiesbaden, Germany); a few cases were assessed by conventional cytology. HPV status was determined using either Hybrid Capture 2 test (Qiagen, Hilden, Germany) or Abbott RealTime High Risk HPV test (Abbott Molecular, Wiesbaden, Germany). The majority of biopsy specimens were obtained using cervical biopsy forceps. If more than one biopsy was obtained or if the conization specimens showed different stages of dysplasia, the highest grade of dysplastic change was recorded.

\section{Statistical analysis}

To analyze the extent of correlation between biopsy specimens and the conization results, all results in which the histology of the biopsy was the same grade as or higher than that of the conization specimen were interpreted as being equivalent. This was done in some cases because the biopsy had already removed the higher grade dysplasia and the final histology result would therefore have been inappropriately low. All data were collected and analyzed using Excel Version 2010 (Microsoft Corp., Redmond, USA) and IBM SPSS Statistics 22 (IBM Corporation, Armonk, NY, USA). Testing for significance was done using analysis of variance (ANOVA). A p-value $<0.05$ was considered significant.

\section{Results}

The data of 593 patients were included in the analysis. The mean patient age was 35.7 years (range: $18.1-84.5$ ), and $87.9 \%$ of women were HPV-positive. Detailed patient characteristics are given in - Table 1. The average time between preoperative examination with biopsy and conization was 57 days. One or more biopsies were obtained preoperatively from 580 patients, while 13 patients had only ECC. 


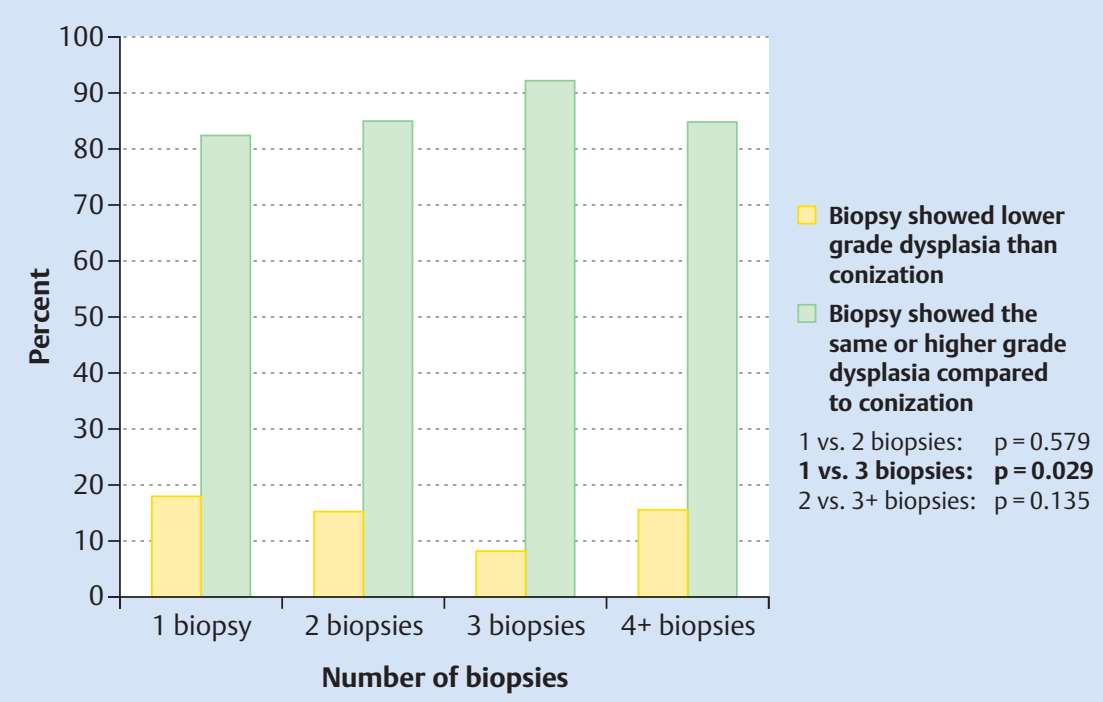

Fig. 1 Agreement between the histological results of the biopsy and the results after conization according to the number of biopsies taken; the accuracy after 3 biopsies was significantly higher compared to the results of only 1 biopsy $(p=0.029)$.

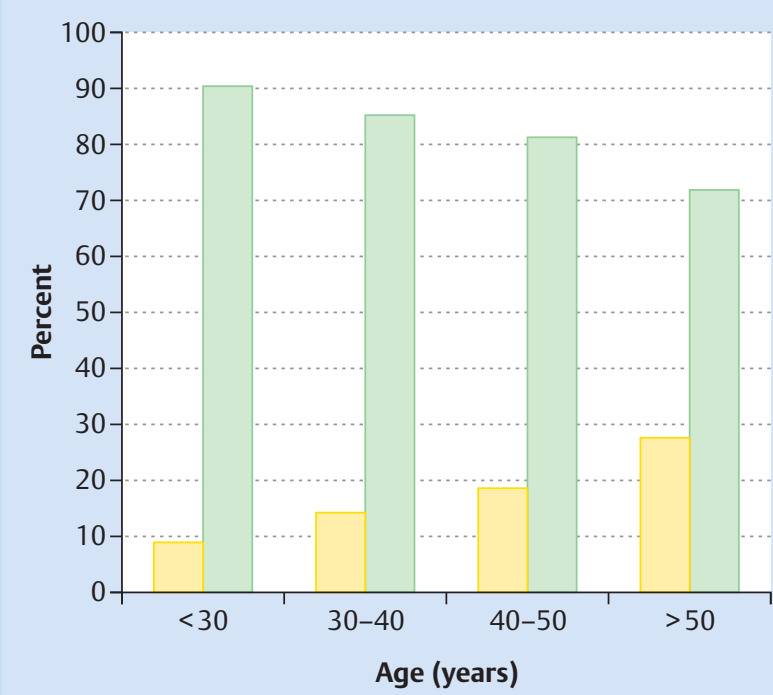

Fig. 2 Agreement between the histological results of the biopsy and the results after conization correlated with patient age. Accuracy decreased significantly as patient age increased $(p=0.008)$.

Biopsy showed lower grade dysplasia than conization

Biopsy showed the same or higher grade dysplasia compared to conization

Table 1 Patient characteristics.

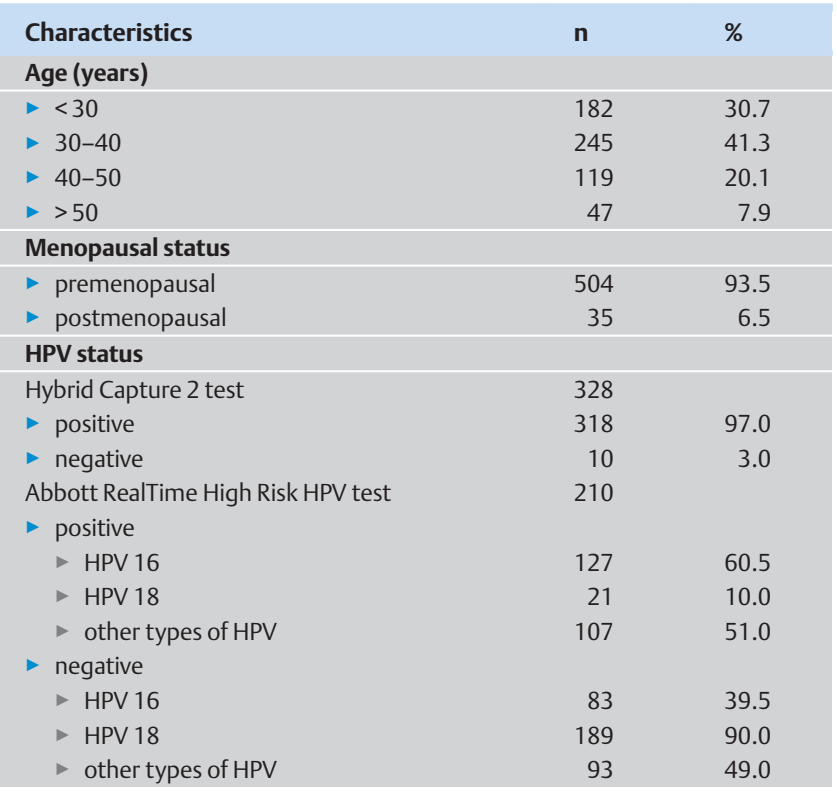

\section{Biopsy collection}

The overall agreement between the results obtained after biopsy and the final histological results was $85.5 \%$. The correlation between histology results and biopsy results was compared and the number of biopsies taken were also factored in. Only one biopsy was taken in 174 cases; two biopsies were taken in 256 cases; three biopsies were taken in 111 cases, and four or more biopsies were taken in 39 cases. The accuracy was higher when more than one biopsy was obtained compared to the results for only one biopsy. When 3 biopsies were taken, the accuracy was significantly higher compared to the results for only one biopsy (91.9 vs. 82.2\%; $\mathrm{p}=0.029$; $\odot$ Fig. 1 ).

The agreement between the final results and the biopsy results correlated significantly with patient age ( $p=0.008$; $\odot$ Fig. 2 ). The highest biopsy correlation of $90.7 \%$ was found in the group of patients aged under 30 years; the lowest correlation was in the group of patients aged over 50 years, only reaching $72.1 \%$. Patients in this age group ( $>50$ years) presented most often with a type 3 transformation zone and were most likely to be postmenopausal $(\mathrm{p}<0.001)$.

The study also looked at the impact of the experience of the examiner who carried out the colposcopy and found no significant 


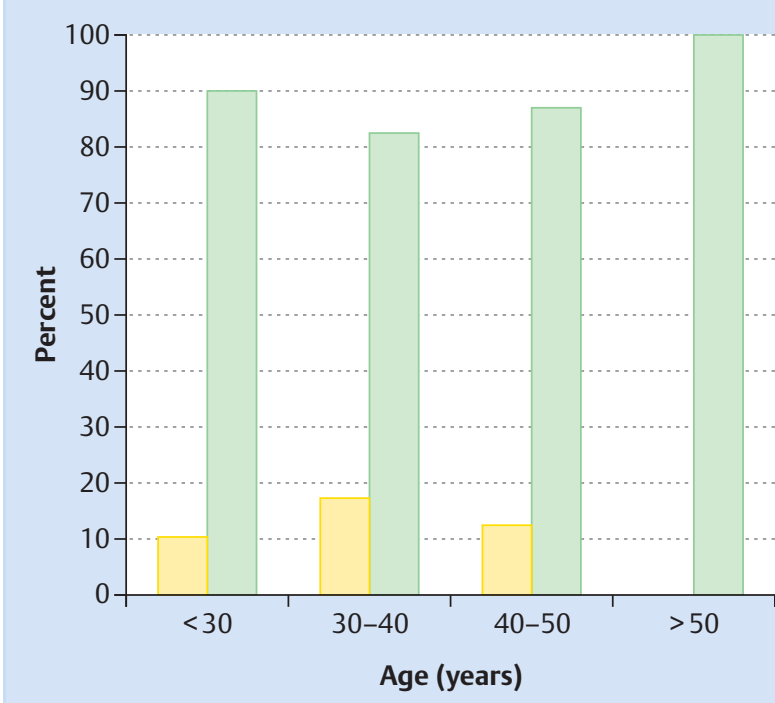

Fig. 3 Agreement between differential cytology and the results after conization depending on patient age. Accuracy was significantly higher for patients aged more than 50 years $(p=0.035)$.

Cytology showed lower grade dysplasia than conization

Cytology showed the same or higher grade dysplasia compared to conization difference in outcomes (results not shown) between examiners (assistant physicians and specialists). Examiners with less experience ( 1 to 2 years) had similar rates of correlation between histological findings to those of examiners with moderate (3 to 5 years) or examiners with extensive experience (more than 5 years). A comparison between the group of examiners who carried out more than 20 preoperative biopsies during the period analyzed in the study and the group of examiners who carried out fewer than 20 biopsies in the same period also showed no significant differences, even when the number of biopsies taken was included in the analysis.

\section{Cytology}

The agreement between the findings of preoperative cytology (cytological findings are the same or higher) with the histological results for the conization was $86.7 \%$ (Pap IIID = CIN 1/2; Pap IVa/ $\mathrm{IVb}=$ CIN 3; Pap V = carcinoma). The accuracy of cytology was significantly correlated with patient age $(p=0.035)$. The correlation increased with increasing patient age and was highest for patients aged over 50 years ( Fig. 3). No correlation was found for the transformation zone.

\section{Endocervical curettage}

ECC was carried out in a total of 169 (28.5\%) patients. The agreement between ECC (results for ECC are the same or higher) and the final histology results was $49.1 \%$. In 19 cases the samples obtained were not representative ( Table 2 ). There was no significant correlation between the accuracy of ECC and menopausal status or transformation zone. Thirteen women were examined only with ECC preoperatively; $83.3 \%$ of them had a type 3 transformation zone. The agreement between ECC and the final histology results was $69.2 \%$ in these cases where only ECC was done.

\section{Discussion}

\section{$\nabla$}

In our study, the agreement between biopsy findings and the final histological results after conization was $85.5 \%$ which is significantly higher than the correlation reported in other studies [3, 5-9]. Those studies predominantly recorded whether there was a precise agreement between biopsy and conization results; they
Table 2 Cytological and histological findings.

\begin{tabular}{|c|c|c|}
\hline Findings & $\mathbf{n}$ & $\%$ \\
\hline Cytology & 563 & \\
\hline • Pap II & 10 & 1.8 \\
\hline > Pap IIw/k & 7 & 1.2 \\
\hline - Pap III & 8 & 1.4 \\
\hline - Pap IIID & 142 & 25.2 \\
\hline - Pap IVa & 378 & 67.1 \\
\hline - Pap IVb & 10 & 1.8 \\
\hline - Pap V & 6 & 1.1 \\
\hline Histology (biopsy) & 580 & \\
\hline - No dysplasia & 23 & 4.0 \\
\hline - CIN 1 & 32 & 5.5 \\
\hline - CIN 2 & 108 & 18.6 \\
\hline$=\mathrm{CIN} 3$ & 402 & 69.3 \\
\hline - AIS & 10 & 1.7 \\
\hline$-C A$ & 3 & 0.5 \\
\hline - Other & 2 & 0.3 \\
\hline Histology (ECC) & 169 & \\
\hline - No dysplasia & 68 & 40.2 \\
\hline - CIN 1 & 11 & 6.5 \\
\hline - CIN 2 & 22 & 13.0 \\
\hline - CIN 3 & 49 & 29.0 \\
\hline - Tissue could not be evaluated & 19 & 11.2 \\
\hline Histology (conization) & 593 & \\
\hline - No dysplasia & 23 & 3.9 \\
\hline - CIN 1 & 40 & 6.7 \\
\hline - CIN 2 & 121 & 20.4 \\
\hline - CIN 3 & 378 & 63.7 \\
\hline - AIS & 9 & 1.5 \\
\hline - CA & 19 & 3.2 \\
\hline - Other & 3 & 0.5 \\
\hline
\end{tabular}

might have reported a higher correlation if overestimated results had been grouped together with precise agreement. The results of another German study were very similar to ours (85.8\%) with regard to histological agreement [10]. Baldauf et al. also reported a very high agreement (89.6\%) between biopsy and conization results (overestimation 4.6\%) [11].

Taking several biopsies appears to have a positive effect on the agreement between biopsy and conization results. Taking three 
biopsies was found to result in a significantly better correlation compared to taking only one biopsy. This assumption is also borne out by other studies which have showed increased sensitivity when several biopsies are taken compared to when only one biopsy is obtained [15]. The highest sensitivity for the detection of high-grade dysplasia as reported in a study by Wentzensen et al. was 95.6\% after taking three biopsies; the figure dropped to $85.6 \%$ after two biopsies and to $60.6 \%$ if only one biopsy was taken [20]. Another study only showed an increase in sensitivity after taking a second biopsy, with no further improvements reported after taking a third biopsy [6].

Factors affecting the number of biopsies taken include the size and the overall impression of the lesions obtained during colposcopy. Well-demarcated, small lesions are more likely to result in only one biopsy being taken. When multifocal lesions are present, the assumption is that more biopsies will be taken, resulting in a greater accuracy [16]. The proposal that randomized biopsies should be routinely taken from all four quadrants of the cervix has been repeatedly discussed, as it has been suggested that this would improve the diagnostic accuracy for low-grade and demarcated small lesions [21]. The diagnostic accuracy varies greatly when the diagnosis is only based on randomized biopsies, ranging from $1.2 \%$ [15] and $2.8 \%$ in cases with cytologically proven low-grade dysplasia to $17.6 \%$ in cases with higher grade dysplasia [17]. However, out of 593 patients included in the study, randomized biopsies from all four quadrants were only taken in seven patients (1.2\% of cases); such limited data do not permit any statements to be made with regard to the benefits of taking randomized biopsies from all four quadrants.

The agreement between biopsy findings and conization results does not appear to depend on the examiner's experience. A study by the ASCUS LSIL Triage Study (ALTS) group reported similar results with regard to the sensitivity of colposcopically guided biopsies [16]. In their study, Baum et al. also compared the agreement between the impression obtained during colposcopy and the histology results of the biopsied specimens and correlated this with the experience of the examiners. They found that when the examiners were assistant physicians in the 2nd year of their specialist training, colposcopy and biopsy results agreed in $77 \%$ of cases; the figure for assistant physicians in the 3rd and 4th year of specialist training was 75 and $73 \%$, respectively. Specially trained nursing staff had a significantly higher rate of agreement of $92 \%$. The authors cited the fact that nursing staff often also carried out the follow-up examinations, which allowed them to develop a better sense of their own accuracy, as a possible reason for this significant difference [22].

The agreement between biopsy findings and results after conization was significantly affected by patient age. The correlation between biopsy and histology decreased with increasing age (highest agreement for patients $<30$ years; lowest agreement in patients aged $>50$ years). Other studies have reported similar findings, with a lower rate of agreement for patients aged $>50$ years [11]. Patients in the age group $>50$ years are more likely to be postmenopausal and have a type 3 transformation zone, which results in a higher rate of colposcopies which are difficult to assess and a lower rate of agreement between the diagnosis based on colposcopy and the histology results [23]. In contrast to these studies, Zuchner et al. described the opposite finding, with the sensitivity of biopsies increasing as patient age increased [6].

The agreement between preoperative cytology and histology results after conization was $86.7 \%$, which is similar to the agreement between biopsies and final histology, and was thus signifi- cantly higher than that reported in other studies $[8,12,13]$. The cytology correlation depends significantly on age and was found to offer better results for patients aged more than 50 years (agreement of $100 \%$ for this age group). However, when considering the accuracy of cytology in our study it is important to remember that the reported rate was for differential cytology, i.e., when the cytologist viewed the cells obtained from cytology, the cytologist was already aware whether the colposcopy was suspicious for higher grade dysplasia. The use of liquid-based cytology for the cytological examination of most patients included in this study tended to slightly delay the creation of specimen slides for cytological assessment, meaning that the histological findings were already known at the time of the cytological assessment, which could have influenced the cytologist. Overall, the rate of abnormal findings was significantly higher compared to screening cytology.

One possible explanation for the superiority of cytology compared to colposcopically guided biopsy in the group aged $>50$ years could be the higher rate of colposcopies which could not be adequately assessed in this age group [24]. If a transformation zone is not visible or only partially visible on colposcopy, the examiner may miss areas where changes have occurred and these changes will not be biopsied. If done correctly, (endocervical) cytology should be able to capture existing cellular changes.

The agreement between preoperative findings and final histology was only $49.1 \%$ for ECC. In contrast to colposcopically guided biopsies or cytological examinations, ECC has only a limited role in the routine diagnosis of intracervical neoplasia. A comparison of other studies showed similar findings with respect to the role played by the different methods in the diagnosis of intracervical neoplasia. A study by Pretorius et al. showed that a diagnosis of CIN 3+ was obtained after biopsy in $63.5 \%$ of cases, and after ECC in $10.8 \%$ of cases. In the isolated group of patients with carcinoma the diagnosis was made after biopsy in $87.1 \%$ of cases [21]. In another study, a diagnosis of CIN 2+ was made using ECC alone instead of biopsy only in $4 \%$ of cases. The overall benefit of ECC was low, although it was found to be slightly higher for patients aged more than 40 years compared to younger patients [25].

The material obtained from ECC is not always suitable for assessment. When all ECC procedures were assessed, it was found that the material obtained from curettage was not usable in a considerable number $(11.2 \%)$ of cases. No dysplasia was detected in $40.2 \%$ of cases. The correlation was not higher for patients with a type 3 transformation zone or patients who were postmenopausal where the likelihood was greater that findings at colposcopy would not be suitable for assessment. However, this finding is limited by the small number $(n=29)$ of women whose examination reports included the transformation zone. Other studies have shown a higher benefit of ECC in this patient population $[15,26]$. The accuracy was higher $(69.2 \%)$ for the 13 cases investigated preoperatively with ECC alone. The majority of patients in this population had a type 3 transformation zone. ECC was only done in 169 cases (28.5\%) of all the patients included in the study, which could limit the validity of the results.

The main weakness of this study is its retrospective nature. If this had been a prospective study, the cytology assessment could have been done with the examiner blinded to the nature of other findings. However, our approach allowed us to include a high number of cases and to define a reliable histological reference using the conization results. In a prospective study it would not be ethically justifiable to use conization as a histological reference point. 


\section{Conclusion for Clinical Practice}

\section{$\nabla$}

Overall, it was found that colposcopy, particularly when combined with three biopsies, is highly accurate for the diagnosis of cervical intraepithelial neoplasia and that this approach leads to highly significant results, particularly for patients under the age of 50 years. Differential cytology is highly accurate (when the histology is partly known) for diagnosing CIN and can be particularly useful for patients over the age of 50 years. The accuracy of ECC is significantly lower than that of colposcopically guided biopsy or cytology but can provide additional information when colposcopy findings are ambiguous (for example, when a type 3 transformation zone is present).

\section{Conflict of Interest}

P. Hillemanns received research grants from GSK, Abbott, and lecture fees from SPMSD, Roche, Hologic. M. Jentschke received speaking fees and partial reimbursement of travel costs from Abbott Molecular, Wiesbaden.

\section{References}

1 Broutet $N$. WHO guidelines for treatment of cervical intraepithelial neoplasia 2-3 and adenocarcinoma in situ. Geneva: WHO; 2014

$2 \mathrm{Kuhn} W$. Colposcopy in the diagnosis of early cervical cancer. Pathologe 2011; 32: 497-504

3 Boicea A, Patrascu A, Surlin V et al. Correlations between colposcopy and histologic results from colposcopically directed biopsy in cervical precancerous lesions. Rom J Morphol Embryol 2012; 53 (3 Suppl.): 735-741

4 Kierkegaard O, Byralsen C, Hansen KC et al. Association between colposcopic findings and histology in cervical lesions: the significance of the size of the lesion. Gynecol Oncol 1995; 57: 66-71

5 Barker B, Garcia F, Lozevski J et al. The correlation between colposcopically directed cervical biopsy and loop electrosurgical excision procedure pathology and the effect of time on that agreement. Gynecol Oncol 2001; 82: 22-26

6 Zuchna C, Hager M, Tringler B et al. Diagnostic accuracy of guided cervical biopsies: a prospective multicenter study comparing the histopathology of simultaneous biopsy and cone specimen. Am J Obstet Gynecol 2010; 203: 321.e1-321.e6

7 Kirkup W, Hill AS. The accuracy of colposcopically directed biopsy in patients with suspected intraepithelial neoplasia of the cervix. $\mathrm{Br}$ J Obstet Gynaecol 1980; 87: 1-4

8 Heatley MK, Bury JP. The correlation between the grade of dyskaryosis on cervical smear, grade of cervical intraepithelial neoplasia (CIN) on punch biopsy and the final histological diagnosis on cone biopsies of the cervix. Cytopathology 1998; 9: 93-99
9 Stubbe M, Broschewitz U, Kramm G et al. [Analysis of conizations performed 2005-2009 in Rostock]. Geburtsh Frauenheilk 2011; 71: 187193

10 Duesing N, Schwarz J, Choschzick M et al. Assessment of cervical intraepithelial neoplasia (CIN) with colposcopic biopsy and efficacy of loop electrosurgical excision procedure (LEEP). Arch Gynecol Obstet 2012; 286: 1549-1554

11 BaldaufJJ, Dreyfus M, Ritter J et al. An analysis of the factors involved in the diagnostic accuracy of colposcopically directed biopsy. Acta Obstet Gynecol Scand 1997; 76: 468-473

12 Yeoh GP, Chan KW. The accuracy of Papanicolaou smear predictions: cytohistological correlation of 283 cases. Hong Kong Med J 1997; 3: 373-376

13 Loiudice L, Abbiati R, Boselli F et al. Improvement of Pap smear sensitivity using a visual adjunctive procedure: a co-operative Italian study on speculoscopy (GISPE). Eur J Cancer Prev 1998; 7: 295-304

14 Rose JD, Byun SY, Sims SM et al. The utility of endocervical curettage: does routine ECC at the time of colposcopy for low-grade cytologic abnormalities improve diagnosis of high-grade disease? Am J Obstet Gynecol 2012; 206: 530.e1-530.e3

15 Nakamura Y, Matsumoto K, Satoh T et al. Optimizing biopsy procedures during colposcopy for women with abnormal cervical cancer screening results: a multicenter prospective study. Int J Clin Oncol 2015; 20: 579-585

16 Gage JC, Hanson VW, Abbey Ket al. Number of cervical biopsies and sensitivity of colposcopy. Obstet Gynecol 2006; 108: 264-272

17 Pretorius RG, Zhang WH, Belinson JL et al. Colposcopically directed biopsy, random cervical biopsy, and endocervical curettage in the diagnosis of cervical intraepithelial neoplasia II or worse. Am J Obstet Gynecol 2004; 191: 430-434

18 Soost HJ. The Munich nomenclature. Recent Results Cancer Res 1993; 133: 105-111

19 Richart RM. Cervical intraepithelial neoplasia. Pathol Annu 1973; 8: 301-328

20 Wentzensen N, Walker JL, Gold MA et al. Multiple biopsies and detection of cervical cancer precursors at colposcopy. J Clin Oncol 2015; 33: 8389

21 Pretorius RG, Belinson JL, Burchette RJ et al. Regardless of skill, performing more biopsies increases the sensitivity of colposcopy. J Low Genit Tract Dis 2011; 15: $180-188$

22 Baum ME, Rader JS, Gibb RK et al. Colposcopic accuracy of obstetrics and gynecology residents. Gynecol Oncol 2006; 103: 966-970

23 Cheng X, Feng $Y$, Wang X et al. The effectiveness of conization treatment for post-menopausal women with high-grade cervical intraepithelial neoplasia. Exp Ther Med 2013; 5: 185-188

24 Benedet JL, Anderson GH, Boyes DA. Colposcopic accuracy in the diagnosis of microinvasive and occult invasive carcinoma of the cervix. Obstet Gynecol 1985; 65: 557-562

25 Solomon D, Stoler M, Jeronimo J et al. Diagnostic utility of endocervical curettage in women undergoing colposcopy for equivocal or low-grade cytologic abnormalities. Obstet Gynecol 2007; 110 (2 Pt 1): 288-295

26 Massad LS, Collins YC. Using history and colposcopy to select women for endocervical curettage. Results from 2,287 cases. J Reprod Med 2003; 48: 1-6 